
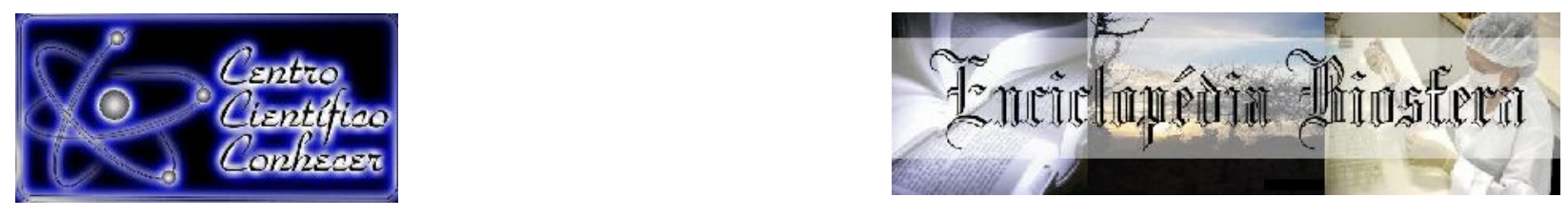

\title{
BIOINDICADORES DE QUALIDADE DE SOLO SOB CULTIVO DO CAFEEIRO EM FUNÇÃO DA ADUBAÇÃO CONVENCIONAL E ORGÂNICA
}

Gustavo Moreira Ribeiro ${ }^{1}$, Edmar Isaias de Melo $^{2}$, Marco lony dos Santos Fernandes $^{3}$, Deivid da Silva Gallet ${ }^{4}$, Gleice Aparecida de Assis ${ }^{5}$

${ }^{1}$ Bacharel em Agronomia pela Universidade Federal de Uberlândia (UFU), Monte CarmeloMG, Brasil.

E-mail: gustavo.m.01@hotmail.com

${ }^{2}$ Professor Doutor do curso de Agronomia pela Universidade Federal de Uberlândia (UFU), Monte Carmelo-MG, Brasil.

${ }^{3}$ Discente de Agronomia pela Universidade Federal de Uberlândia (UFU), Monte CarmeloMG, Brasil.

${ }^{4}$ Discente de Agronomia pela Universidade Federal de Uberlândia (UFU), Monte CarmeloMG, Brasil.

${ }^{5}$ Professora Doutora do curso de Agronomia da Universidade Federal de Uberlândia (UFU), Monte Carmelo-MG, Brasil.

Recebido em: 15/02/2020 - Aprovado em: 15/03/2020 - Publicado em: 30/03/2020 DOI: 10.18677/EnciBio_2020A13

\begin{abstract}
RESUMO
A crescente demanda por cafés especiais, priorizando qualidade do produto e sustentabilidade ambiental vem impulsionando a produção orgânica do cafeeiro e aumentado o interesse pela avaliação de bioindicadores de qualidade do solo mensurando os efeitos dos sistemas de manejo. O objetivo deste trabalho foi avaliar parâmetros de bioindicação de qualidade de solo em cafeeiros em função da adubação orgânica e convencional, tendo solo de mata nativa como referência. $O$ experimento ocorreu em Monte Carmelo - MG, com a cultivar Catucaí Amarelo 20/15 cv 479 em delineamento em blocos casualizados com cinco blocos. Os tratamentos consistiram na aplicação de composto orgânico e o fertilizante orgânico líquido via "drench" e o tratamento convencional da fazenda (testemunha). Foram coletadas três subamostras por parcela experimental na projeção da copa do cafeeiro e em área de mata nativa próxima ao experimento, resultando em uma amostra composta por parcela de cada tratamento. Foram avaliados os parâmetros $\mathrm{pH}$, condutividade elétrica, respiração basal do solo, carbono lábil, fósforo assimilável e nitrogênio amoniacal. O solo sob adubação orgânica proporcionou teores de nitrogênio amoniacal e fósforo assimilável maior que o sistema convencional. Os valores de carbono lábil foram semelhantes em ambos os solos. A condutividade elétrica deve ser monitorada constantemente no solo sob cultivo para evitar a elevação a níveis tóxicos para o cafeeiro. De modo geral, o solo sob adubação orgânica não contribuiu para melhoria da atividade biológica, mas manteve ou elevou os valores de fósforo e $\mathrm{C}_{\text {lábil. }}$
\end{abstract}

PALAVRAS-CHAVE: bioindicação de qualidade do solo, cafeicultura orgânica, Coffea arabica L. 


\title{
SOIL QUALITY BIOINDICATORS UNDER COFFEE GROWING DUE TO CONVENTIONAL AND ORGANIC FERTILIZATION
}

\begin{abstract}
The growing demand for specialty coffees, prioritizing product quality and environmental sustainability has promoted organic coffee production and increased interest in the assessment of soil quality bioindicators measuring the effects of management systems. The aim of this work is to evaluate parameters of soil bioindication quality in coffee plants due to organic and conventional fertilization, using native forest soil as a reference. The experiment happened in Monte Carmelo MG, with the cultivar Catucaí Amarelo 20/15 cv 479 in a randomized block designed with five blocks. The treatments consisted in the application of organic compost and liquid organic fertilizer via "drench and the conventional treatment of the farm (spectator). Three subsamples per experimental plot were collected in the projection from the top of the coffee and in an area of native forest close to the experiment, resulting in a sample composed of each treatment plot. The parameters $\mathrm{pH}$, electrical conductivity, basal soil respiration, labile carbon, assimilable phosphorus and ammoniacal nitrogen were evaluated. The soil under organic fertilization provided levels of ammoniacal nitrogen and assimilable phosphorus higher than the conventional system, however ammoniacal nitrogen was lower than the soil under native vegetation, for labile carbon were similar in both soils. Regarding electrical conductivity, they must constantly monitor the soil under cultivation to avoid elevation to toxic levels for the coffee plant. In general, the soil under organic fertilization does not contribute to the improvement of biological activity, but maintained or increased values of phosphorus and Clábil.
\end{abstract}

KEYWORDS: soil quality bioindication, organic coffee, Coffea arabica L.

\section{INTRODUÇÃO}

O Brasil é o maior produtor e exportador mundial de café, sendo responsável por aproximadamente $37 \%$ de todo o café produzido no mundo. Minas Gerais é o estado que possui maior área de cafeeiro arábica no Brasil, com 1,226 milhão de hectares, ocupando $69,8 \%$ da área cultivada com café no país, respondendo por $51,7 \%$ na estimativa da produção nacional (CONAB, 2020).

O movimento mais atual da cafeicultura é marcado pela revolução no mercado de cafés especiais, devido à mudança na percepção dos consumidores e pela adoção de fatores de diferenciação do produto. Essas mudanças teriam sido promovidas pelo aumento da demanda de cafés de qualidade, oriundos de origens específicas, diversificação na forma de comercialização da bebida, maior atenção à sustentabilidade ambiental e às vulnerabilidades dos cafeicultores (BORRELLA et al., 2015).

Os sistemas de produção orgânica têm ganhado espaço promovendo uma agricultura mais sustentável e a conservação dos ecossistemas, objetivando viabilidade econômica, devido a diferenciação do produto atender o mercado de cafés orgânicos e especiais, o qual tem se mostrado mais lucrativo que a comercialização por commodity (LOPES et al., 2014)

Produtores normalmente utilizam análises químicas para avaliação da qualidade do solo, porém, com a diversificação dos sistemas de cultivos e possibilidade de serviços ambientais e certificação de fazendas tem crescido o 
interesse por atributos que avaliem a qualidade do solo e a sustentabilidade dos sistemas de cultivos como forma de avaliar as práticas agrícolas. Dessa forma, tem aumentado o interesse pela avaliação de parâmetros de bioindicação de qualidade do solo (MENDES et al., 2015).

Para avaliar a sustentabilidade de um sistema de cultivo é importante utilizar bons indicadores de qualidade do solo (AZEVEDO JUNIOR et al., 2017). A qualidade do solo pode ser mensurada por indicadores do ponto de vista ecológico, econômico ou social e levam em consideração certa propriedade ou função do solo que tenha correlação com as mudanças em um agroecossistema ou sistema florestal (MARTINEZ-SALGADO et al., 2010).

Os indicadores de qualidade de solo têm sido empregados para avaliação dos impactos da atividade agrícola. Eles podem representar a qualidade física, química e biológica do solo e estão diretamente relacionados aos processos do solo. Alguns atributos como pH, salinidade, carbono orgânico, fósforo assimilável e CTC, possibilitam o entendimento da dinâmica dos nutrientes e a relação com a fertilidade do solo. Indicadores biológicos fornecem um indicativo da microbiota do solo, a qual se relaciona diretamente com processos de decomposição, formação da matéria orgânica recalcitrante, ciclagem de nutrientes, atividade enzimática e microbiana Os bioindicadores de qualidade do solo têm a capacidade de responder às mudanças no solo, por exemplo, a avaliação da atividade microbiana têm sido amplamente utilizada devido à relação com o tipo de cobertura vegetal e sustentabilidade dos ecossistemas, sendo utilizado como indicador sensível de estresse ecológico ou dos processos de restauração do solo em ambientes naturais e nos agroecossistemas sob cultivo (SILVA et al., 2015a).

Portanto, parâmetros de bioindicação de qualidade do solo têm se mostrado uma importante ferramenta para avaliar e comparar o efeito dos diferentes sistemas de manejo empregados sob a qualidade do solo. Nesse sentido objetivou-se com este trabalho avaliar a qualidade de solo, por meio de parâmetros de bioindicação, em área sob cultivo do cafeeiro em função da adubação orgânica e convencional, tendo solo de mata nativa como referência.

\section{MATERIAL E MÉTODOS}

O ensaio foi conduzido na Fazenda Araras 2 (1843’02,0"S 47³2'49,1"W), município de Monte Carmelo-MG, localizado na microrregião do Triângulo Mineiro e Alto Paranaíba, a $886 \mathrm{~m}$ de altitude. O solo da área experimental é classificado como LATOSSOLO VERMELHO.

O plantio da lavoura de C. arabica L, cultivar Catucaí Amarelo 20/15 cv 479, foi efetuado em dezembro de 2016 e a diferenciação dos tratamentos foi realizada em dezembro de 2017. A lavoura é irrigada via gotejamento, com gotejadores espaçados em $0,70 \mathrm{~m}$ e vazão $2,6 \mathrm{~L} \mathrm{ha}^{-1}$. O espaçamento de plantio é de 3,8 $\mathrm{m}$ entre linhas por $0,6 \mathrm{~m}$ entre plantas. $\mathrm{O}$ solo foi analisado quanto às características químicas nas camadas de 0-20 cm no ano de 2017 e de 0-20 cm e 0-40 cm em janeiro de 2018 (Quadro 1). 
QUADRO 1- Caracterização química do solo na camada de 0-20 e 20-40 cm da área experimental na Fazenda Araras 2 no município de Monte Carmelo-MG

\begin{tabular}{lrrr}
\hline \multicolumn{1}{c}{ Atributos químicos do solo } & $\mathbf{2 0 1 7}$ & \multicolumn{2}{c}{$\mathbf{2 0 1 8}$} \\
\cline { 2 - 4 } & $\mathbf{0 - 2 0}$ & $\mathbf{0 - 2 0}$ & $\mathbf{0 - 4 0}$ \\
\hline $\mathrm{pH} \mathrm{em} \mathrm{H} \mathrm{H}_{2} \mathrm{O}$ & 4,20 & 5,80 & 6,30 \\
$\mathrm{P}\left(\mathrm{mg} \mathrm{dm}^{-3}\right)$ & 18,00 & 102,50 & 124,80 \\
$\mathrm{~K}^{+}\left(\mathrm{cmol}_{\mathrm{c}} \mathrm{dm}^{-3}\right)$ & 0,42 & 0,52 & 0,46 \\
$\mathrm{Ca}^{+2}\left(\mathrm{cmol}_{\mathrm{c}} \mathrm{dm}^{-3}\right)$ & 1,20 & 6,60 & 6,60 \\
$\mathrm{Mg}^{+2}\left(\mathrm{cmol}_{\mathrm{c}} \mathrm{dm}^{-3}\right)$ & 0,22 & 1,00 & 1,00 \\
$\mathrm{Al}^{+3}\left(\mathrm{cmol}_{\mathrm{c}} \mathrm{dm}^{-3}\right)$ & 0,62 & 0,00 & 0,00 \\
$\mathrm{H}^{++} \mathrm{Al}^{+3}\left(\mathrm{cmol}_{\mathrm{c}} \mathrm{dm}^{-3}\right)$ & 7,20 & 2,90 & 2,40 \\
$\mathrm{SB}\left(\mathrm{cmol}_{\mathrm{c}} \mathrm{dm}^{-3}\right)$ & 1,80 & 8,10 & 8,10 \\
$\mathrm{~V}(\%)$ & 20,00 & 74,00 & 77,00 \\
$\mathrm{~m}(\%)$ & 25,00 & 0,00 & 0,00 \\
$\mathrm{CTC}\left(\mathrm{cmol}_{\mathrm{c}} \mathrm{dm}^{-3}\right)$ & 2,40 & 8,10 & 8,10 \\
$\mathrm{Zn}\left(\mathrm{mg} \mathrm{dm}^{-3}\right)$ & 3,70 & 2,50 & 2,60 \\
$\mathrm{Mn}\left(\mathrm{mg} \mathrm{dm}^{-3}\right)$ & 4,50 & 4,10 & 4,10 \\
$\mathrm{Fe}\left(\mathrm{mg} \mathrm{dm}^{-3}\right)$ & 5,70 & 24,00 & 24,00 \\
$\mathrm{Cu}\left(\mathrm{mg} \mathrm{dm}^{-3}\right)$ & 6,80 & 2,90 & 2,70 \\
$\mathrm{~B}\left(\mathrm{mg} \mathrm{dm}^{-3}\right)$ & 0,62 & 0,60 & 0,57 \\
$\mathrm{SSO}{ }_{4}^{-2}\left(\mathrm{mg} \mathrm{dm}^{-3}\right)$ & 93,00 & 227,00 & 198,00 \\
$\mathrm{MO}\left(\mathrm{dag} \mathrm{ha}^{-1}\right)$ & 3,20 & 3,50 & 3,30 \\
\hline $\mathrm{Análise} \mathrm{realizadas} \mathrm{no}$ & & &
\end{tabular}

Análises realizadas no Laboratório Brasileiro de Análises Agrícolas (LABRAS).

Para condução do experimento foi utilizado o composto orgânico, proveniente do tratamento de materiais orgânicos de origem vegetal por meio da compostagem natural sem adição de agentes aceleradores (Quadro 2) e o fertilizante orgânico líquido (Quadro 3).

QUADRO 2- Caracterização do composto orgânico

\begin{tabular}{lc}
\hline \multicolumn{1}{c}{ Atributos } & Resultados \\
\hline $\mathrm{N}$ & $1,20 \%$ \\
$\mathrm{P}_{2} \mathrm{O}_{5}$ & $1,60 \%$ \\
$\mathrm{~K}_{2} \mathrm{O}$ & $0,93 \%$ \\
$\mathrm{Ca}$ & $4,50 \%$ \\
$\mathrm{Mg}$ & $0,42 \%$ \\
$\mathrm{~S}$ & $0,55 \%$ \\
$\mathrm{~B}$ & $0,0002 \%$ \\
$\mathrm{Cu}$ & $0,009 \%$ \\
$\mathrm{Fe}$ & $1,20 \%$ \\
$\mathrm{Mn}$ & $0,07 \%$ \\
$\mathrm{Zn}$ & $0,008 \%$ \\
$\mathrm{SiO}$ & $43,10 \%$ \\
$\mathrm{Carbono}$ orgânico total & $14,50 \%$ \\
Extrato húmico total & $26,10 \%$ \\
Ácidos húmicos & $17,00 \%$ \\
Ácidos fúlvicos & $9,10 \%$ \\
Umidade a $65^{\circ}$ & $27,6 \%$ \\
pH & 7,40
\end{tabular}


Condutividade elétrica

CTC

Relação CTC/C

Capacidade de retenção de água

Relação C/N

$1,4 \mathrm{mScm}^{-1}$

$312,0 \mathrm{mmolkg}^{-1}$

21,5

$106,0 \%$

8,8

Matéria orgânica

$27,4 \%$

Análise segundo metodologia do Manual de métodos analíticos oficiais para fertilizantes e corretivos (MAPA).

QUADRO 3- Caracterização do fertilizante orgânico líquido.

Atributos

Carbono orgânico

$\mathrm{K}_{2} \mathrm{O}$

Garantias do produto comercial.

O delineamento experimental utilizado foi o de blocos casualizados, com cinco blocos. Cada parcela foi composta por 20 plantas, sendo a área útil composta pelas oito plantas centrais. $\mathrm{O}$ experimento apresentou 25 parcelas (incluindo a mata nativa como referência), sendo cada unidade experimental intercalada com uma linha de bordadura, a fim de evitar a interferência entre os tratamentos. Em todos os tratamentos referentes ao manejo de transição para o orgânico, as adubações foram realizadas de três formas: adubação de cobertura, "drench" e pulverização. No tratamento convencional, o adubo padrão utilizado para cobertura foi o formulado 04-14-8 e para fertirrigação foi mantido o padrão da propriedade (Quadro 5).

Cada tratamento apresentou um intervalo de aplicação (Quadro 4) para adubação de cobertura, "drench" e pulverização. Foram testados quatro tratamentos: adubação de cobertura com $150 \mathrm{~g}_{\text {planta }}{ }^{-1}$ do composto na dose de $1,7 \mathrm{t} \mathrm{ha}^{-1}$ (T1); $300 \mathrm{~g}_{\text {planta }}{ }^{-1}$ na dose de 3,38 tha $\mathrm{t}^{-1}$ (T2); $500 \mathrm{~g}_{\text {planta }}{ }^{-1}$ na dose de 5,6 $\mathrm{t} \mathrm{ha}^{-1}$ (T3) e tratamento convencional da propriedade (T4). Para os tratamentos T1, T2 e T3 foi aplicado fertilizante orgânico liquído via "drench" na dose de $3 \mathrm{ml} \mathrm{L}^{-1}$ e $600 \mathrm{ml}$ de calda planta-1 , adicionado de pulverizações do produto na dose de $3 \mathrm{ml} \mathrm{L}^{-1} \mathrm{H}_{2} \mathrm{O}$. No T4 foi realizada a aplicação de produtos fitossanitários para controle de pragas e doenças e adubação via fertirrigação (Quadro 5) e aplicação de fosfato monoamônico (MAP) e sulfato de amônio para adubação de reposição.

QUADRO 4- Descrição dos tratamentos e intervalos de aplicação em dias

\section{Tratamentos}

Periodicidade

\begin{tabular}{lccc}
\hline $\begin{array}{l}\text { T1 - Composto orgânico + fertilizante } \\
\text { orgânico liquído }\end{array}$ & 90 & 30 & 20 \\
$\begin{array}{l}\text { T2 - Composto orgânico + fertilizante } \\
\text { orgânico liquído }\end{array}$ & 30 & 90 & 30 \\
$\begin{array}{l}\text { T3 - Composto orgânico+ fertilizante } \\
\text { orgânico liquído }\end{array}$ & 30 & 60 & 15 \\
$\begin{array}{l}\text { T4 - Tratamento Convencional } \\
\text { T5-Mata nativa }\end{array}$ & - & - & - \\
\hline
\end{tabular}


QUADRO 5- Ingredientes ativos e nutrientes aplicados no tratamento padrão da fazenda

\begin{tabular}{|c|c|c|}
\hline Ingrediente ativo & $\begin{array}{l}\text { Dose } \\
\left(\text { L ha }^{-1}\right)\end{array}$ & $\begin{array}{l}\text { Volume de } \\
\text { calda }\left(\mathrm{L} \mathrm{ha}^{-1}\right)\end{array}$ \\
\hline Macronutriente $\mathrm{K}$ & 2,0 & 500 \\
\hline Macro e micronutrientes $\mathrm{Ca}$ e $\mathrm{B}$ & 2,0 & 500 \\
\hline Macronutrientes $\mathrm{Ne} \mathrm{P}$ & 0,5 & 500 \\
\hline Macro e micronutrientes $\mathrm{Ca}$ e $\mathrm{B}$ & 1,0 & 500 \\
\hline Azoxistrobina, Estrobilurina & 0,5 & 500 \\
\hline Azoxistrobina, Cicopronazol & 0,75 & 500 \\
\hline Hidróxido de cobre & 3,0 & 500 \\
\hline Azoxistrobina & 2,0 & 500 \\
\hline Abamectina & 0,4 & 500 \\
\hline Abamectina & 1,0 & 500 \\
\hline Abamectina & 0,5 & 500 \\
\hline Propargito & 1,0 & 500 \\
\hline Ingrediente ativo & $\begin{array}{c}\text { Dose } \\
\left(\mathrm{kg} \mathrm{ha}^{-1}\right)\end{array}$ & $\begin{array}{l}\text { Volume de } \\
\text { calda }\left(\mathrm{L} \mathrm{ha}^{-1}\right)\end{array}$ \\
\hline Mono Amônio Fosfato & 25 & 500 \\
\hline Tiofanato-metílico & 1,0 & 500 \\
\hline Micronutrientes & 3,0 & 500 \\
\hline Micronutriente B & 3,0 & 500 \\
\hline Macro e micronutrientes & 2,0 & 500 \\
\hline Tiametoxan & 2,0 & 500 \\
\hline
\end{tabular}

Para análise dos bioindicadores de solo, foram coletadas três subamostras por parcela experimental na projeção da copa do cafeeiro e em uma área de mata nativa próxima ao experimento, com auxílio de um enxadão na profundidade de 0 a $10 \mathrm{~cm}$ que foram homogeneizadas resultando em uma amostra composta por unidade experimental.

A atividade microbiana do solo foi avaliada pela determinação da respiração basal do solo (RBS), cujo procedimento foi realizado conforme metodologia descrita por DIONÍSIO et al. (2016). Inicialmente foi determinada a capacidade de retenção de água (CRA) e posterior correção da umidade para 60,0\% da CRA, com água destilada. A massa referente a 100,00 g de solo úmido, previamente peneirado, em peneira de abertura de 2,00 $\mathrm{mm}$, foi pesada e transferida para um frasco de vidro com tampa hermética. Dentro do frasco de vidro foi colocado um tubo de ensaio contendo $10,0 \mathrm{ml}$ de $\mathrm{NaOH} 0,5 \mathrm{~mol} \mathrm{~L}^{-1}$ padronizado para capturar o $\mathrm{CO}_{2}$ produzido e outro tubo de ensaio contendo $10,0 \mathrm{~mL}$ de água destilada para manter a umidade do ambiente do frasco. Para cada dez frascos de vidro a serem incubados, foi realizada uma prova em branco, que correspondeu a um frasco contendo apenas um tubo de ensaio com $10 \mathrm{~mL}$ de $\mathrm{NaOH} 0,5 \mathrm{~mol} \mathrm{~L}^{-1}$ padronizado e outro contendo $10,0 \mathrm{~mL}$ de água destilada. Os frascos de vidro foram hermeticamente fechados e incubados em temperatura ambiente por uma semana (168 horas). Após o período de incubação, retirou-se dos frascos de vidro os tubos de ensaio contendo $\mathrm{NaOH}$, cuja solução foi transferida para erlenmeyer de $125,0 \mathrm{~mL}$, adicionando $1,0 \mathrm{~mL}$ de $\mathrm{BaCl}_{2}(10 \% \mathrm{~m} / \mathrm{V})$ e duas gotas de fenolftaleína. $\mathrm{O}$ excesso de $\mathrm{NaOH}$ foi titulado com $\mathrm{HCl} \mathrm{0,5} \mathrm{mol.} \mathrm{L}^{-1}$. 
A determinação do $\mathrm{pH}$ foi realizada em duplicata, em solução de cloreto de cálcio $\left(\mathrm{CaCl}_{2}\right)$, e em água. Foram pesadas amostras de $10 \mathrm{~mL}$ de solo acondicionadas em frascos plásticos. Em seguida foram adicionados $25 \mathrm{~mL}$ de $\mathrm{CaCl}_{2}$ a $0,01 \mathrm{~mol} \mathrm{~L}^{-1}$, agitando-se a amostra com bastão de vidro individual e deixando-se em repouso por uma hora. Logo após agitou-se a amostra e o pH foi determinado em medidor de $\mathrm{pH}$ modelo mPA-210 TECNOPON.

Para quantificação da condutividade elétrica, foi utilizada a relação 1:2,5 solo:suspensão ( $10 \mathrm{~g}$ de solo e $25 \mathrm{~mL}$ de água deionizada. As amostras foram agitadas e posteriormente deixadas em repouso até a sedimentação do material em suspensão e clareamento do sobrenadante, fazendo-se em seguida a leitura da condutividade elétrica no sobrenadante com um condutivímetro modelo mCA-100 TECNOPON.

A mensuração do carbono lábil foi realizada em duplicata, segundo Blair et al. (1995), adaptado por (SHANG ; TIESSEN, 1997). Pesou-se 1,0 g de terra fina seca ao ar (TFSA) de peneira de malha menor que $0,5 \mathrm{~mm}$, sendo transferida para um tubo falcon de 50,0 mL envolto com papel alumínio. Foram adicionados $25 \mathrm{~mL}$ da solução de $\mathrm{KMnO}_{4} 0,033 \mathrm{~mol} \mathrm{~L}^{-1}$, agitados por 1 hora, e em seguida centrifugados por 5 minutos. Após centrifugação, $40 \mu \mathrm{L}$ do sobrenadante foi pipetado em tubos de ensaio, completando o volume de $10 \mathrm{~mL}$ com água. Após isso, foi feita a leitura em espectrofotômetro UV-Viz, modelo DR40000, da HACH em comprimento de onda de $565 \mathrm{~nm}$. A [KMnO4] residual após a reação com a fração lábil da matéria orgânica presente no solo, foi determinada por curva de calibração construída a partir de uma solução estoque de $\mathrm{KMnO}_{4}, 0,00060 \mathrm{~m}$ mol. $\mathrm{L}^{-1}$. A mudança na concentração de $\mathrm{KMnO}_{4}$ foi usada para estimar a quantidade de carbono oxidado, assumindo que 1 $\mathrm{mmol} \mathrm{L}^{-1}$ de $\mathrm{KMnO}_{4}$ é consumido na oxidação de $0,75 \mathrm{mmol}$ ou $9 \mathrm{mg}$ de carbono.

Para determinação de fósforo assimilável inicialmente foram adicionados 5 $\mathrm{cm}^{3}$ de solo em erlenmeyer de $125 \mathrm{~mL}$ e $50 \mathrm{~mL}$ de solução extratora $(\mathrm{HCl} 0,05 \mathrm{~mol}$ $\mathrm{L}^{-1}$ e $\mathrm{H}_{2} \mathrm{SO}_{4} \quad 0,0125 \mathrm{~mol} \mathrm{~L}^{-1}$ ). Logo após realizou-se agitação durante 5 minutos no agitador circular horizontal, sendo posteriormente colocada para decantar durante uma noite, tendo antes o cuidado de desfazer os montículos que se formaram no fundo dos erlenmeyers. Pipetou-se um volume do extrato da amostra $(1.000 \mu \mathrm{L}$ para amostra de mata e $300 \mu \mathrm{L}$ para amostra dos tratamentos), definido com base na faixa linear de resposta da curva de calibração, para tubo de ensaio com capacidade de 10,0 mL, no qual foi também adicionado $3,0 \mathrm{~mL}$ de solução ácida de molibdato de amônio diluída e $0,2 \mathrm{~mL}$ de ácido ascórbico $(1,5 \% \mathrm{~m} / \mathrm{V})$ e completou-se o volume até $10,0 \mathrm{~mL}$ com água destilada. Soluções padrões $\left(0 ; 0,5 ; 1,0 ; 1,5\right.$ e $\left.2,0 \mathrm{mg} \mathrm{L}^{-1}\right)$, em triplicata, preparadas a partir de solução estoque de $\mathrm{KH}_{2} \mathrm{PO}_{4}\left(25,0 \mathrm{mg} \mathrm{L}^{-1}\right.$ de $\left.\mathrm{P}\right)$, e adição de 3,0 mL de solução ácida de molibdato de amônio diluída e $0,2 \mathrm{~mL}$ de ácido ascórbico $(1,5 \% \mathrm{~m} / \mathrm{V})$, foram utilizadas para obtenção da curva de calibração. Tubos de ensaio contendo as soluções padrões e as amostras foram agitadas manualmente durante 1 a 2 minutos e foram deixados em repouso por uma hora. Após esse período fez-se a leitura da absorbância, no comprimento de onda de 660 $\mathrm{nm}$, em espectrofotômetro UV-Vis, da HACH, modelo DR 40000.

O íon amônio foi extraído do solo conforme metodologia adaptada de Silva et al. (2010) e a concentração presente no sobrenadante foi determinada pelo método de análise por injeção em fluxo com detecção condutométrica - FIA/CE (PASQUINI; DE FARIA, 1987). 
Os dados obtidos foram submetidos à análise de variância com a aplicação do teste de $F$, a $5 \%$ de probabilidade e as médias dos tratamentos foram comparadas pelo teste de Scott Knott utilizando-se o software SISVAR (FERREIRA, 2011). Foi realizada a transformação $\log x$ para atender à pressuposição de normalidade dos dados para as características nitrogênio amoniacal, fósforo assimilável e condutividade elétrica.

\section{RESULTADOS E DISCUSSÃO}

De acordo com a análise de variância (Tabela 1), foi possível verificar diferenças significativas entre os tratamentos em relação à concentração de nitrogênio amoniacal e fósforo assimilável, respiração basal, condutividade elétrica e pH pelo Teste $\mathrm{F}$ ao nível de $5 \%$ de probabilidade. Para a variável $\mathrm{C}_{\text {Lábil }}$ não houve efeito significativo entre os tratamentos.

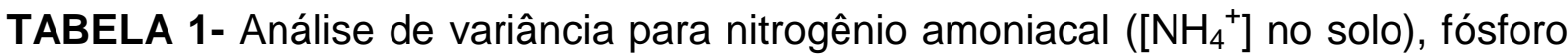
assimilável ([P] no solo), CLábil, respiração basal do solo (RBS), condutividade elétrica $(\mathrm{CE})$ e pH em solo sob manejo de transição para orgânico, convencional e mata nativa

\begin{tabular}{|c|c|c|c|c|c|c|c|}
\hline \multirow[b]{2}{*}{ FV } & \multirow[b]{2}{*}{ GL } & \multicolumn{6}{|c|}{ QM } \\
\hline & & $\begin{array}{c}{\left[\mathrm{NH}_{4}{ }^{+}\right]} \\
\text {no solo }{ }^{1}\end{array}$ & $\begin{array}{l}\text { [P] no } \\
\text { solo }^{1}\end{array}$ & $\mathbf{C}_{\text {Lábil }}$ & RBS & $\mathrm{CE}^{1}$ & $\mathrm{pH}$ \\
\hline Tratamentos & 4 & $0,49 * * *$ & $1,15^{\star *}$ & $1,05^{\mathrm{ns}}$ & $0,06^{*}$ & $0,17^{* *}$ & $1,18^{* *}$ \\
\hline Blocos & 4 & 0,04 & 0,08 & 1,74 & 0,02 & 0,04 & 0,13 \\
\hline Erro & 16 & 0,02 & 0,03 & 0,52 & 0,01 & 0,01 & 0,08 \\
\hline CV (\%) & & 15,90 & 9,92 & 10,36 & 14,24 & 6,03 & 4,86 \\
\hline
\end{tabular}

Para os valores de nitrogênio amoniacal $\left(\mathrm{NH}_{4}^{+}\right)$houve diferença significativa entre solo de mata nativa, manejo de transição para orgânico e convencional. Foram observados maiores valores para mata nativa $\left(24,69 \mathrm{mg} \mathrm{g}^{-1}\right)$, seguido do cafeeiro em transição, com valor médio de $8,36 \mathrm{mg} \mathrm{g}^{-1}$ entre os tratamentos com manejo em transição, os quais não diferiram significativamente entre si, seguido do tratamento convencional, apresentando valor médio de $3,50 \mathrm{mg} \mathrm{g}^{-1} \mathrm{de} \mathrm{NH}_{4}{ }^{+}$no solo (Tabela 2).

TABELA 2- Médias dos valores de nitrogênio amoniacal ( $\left[\mathrm{NH}_{4}{ }^{+}\right]$no solo), fósforo assimilável ([P] no solo), CLábil e respiração basal do solo (RBS) sob manejo de transição para orgânico e convencional comparado com mata nativa

\begin{tabular}{ccccc}
\hline Tratamento & $\begin{array}{c}{\left[\mathbf{N H}_{4}^{+}\right] \mathbf{~ n o ~ s o l o ~}} \\
\left(\mathbf{m g ~ g}^{-1}\right)\end{array}$ & $\begin{array}{c}{[\mathbf{P}] \mathbf{~ n o ~ s o l o}} \\
\left(\mathbf{m g ~ d m}^{-3}\right)\end{array}$ & $\begin{array}{c}\mathbf{C}_{\text {Lábil }} \\
\left(\mathbf{m g ~ g}^{-1}\right)\end{array}$ & $\begin{array}{c}\mathbf{R B S} \\
\left(\mathbf{m g ~ C}-\mathbf{C O}_{\mathbf{~}} \mathbf{~ g ~}^{-1} \mathbf{h}\right)\end{array}$ \\
\hline MATA & $24,69 \mathrm{a}$ & $7,24 \mathrm{~b}$ & $6,42 \mathrm{a}$ & $0,942 \mathrm{a}$ \\
T1 & $8,36 \mathrm{~b}$ & $101,11 \mathrm{a}$ & $7,10 \mathrm{a}$ & $0,666 \mathrm{~b}$ \\
T2 & $9,48 \mathrm{~b}$ & $96,46 \mathrm{a}$ & $7,32 \mathrm{a}$ & $0,794 \mathrm{~b}$ \\
T3 & $9,74 \mathrm{~b}$ & $94,23 \mathrm{a}$ & $6,49 \mathrm{a}$ & $0,706 \mathrm{~b}$ \\
T4 & $3,50 \mathrm{c}$ & $78,42 \mathrm{a}$ & $7,38 \mathrm{a}$ & $0,762 \mathrm{~b}$ \\
\hline CV $(\%)$ & 15,90 & 9,92 & 10,36 & 14,24 \\
\hline
\end{tabular}

Médias seguidas pela mesma letra não diferem significativamente entre si a $5 \%$ de probabilidade pelo teste de Scott-Knott. 
Avaliando uma lavoura de café conilon em Linhares - ES, adubada com compostos orgânicos preparados com capim-elefante e palha de café na proporção 1:1 (v:v) e com capim-elefante, palha de café e cama de frango na proporção 2:1:1 (v:v:v), Silva et al. (2015b) verificaram aumento nos teores de nitrogênio total e estoque de nitrogênio nos tratamentos que receberam adubações orgânicas em comparação com a adubação mineral no segundo ano agrícola. O nitrogênio presente no composto se encontrava na forma orgânica, contribuindo para a manutenção do nutriente por mais tempo no solo se comparado ao adubo mineral.

Em experimento conduzido em Santa Maria - RS, Lorensini et al. (2014) avaliaram por meio de incubação em laboratório a disponibilidade de $\mathrm{N}$ em solo cultivado com vinhedo, com adição de composto orgânico, ureia e ureia revestida com polímeros, ajustado para uma dose de $40 \mathrm{~kg} \mathrm{de} \mathrm{N} \mathrm{ha}^{-1}$, comparados com solo de mata nativa. Verificaram-se maiores teores de $\mathrm{N}_{-} \mathrm{NH}_{4}{ }^{+}$em solo de mata a partir de 70 dias de incubação, provavelmente devido à mineralização dos compostos orgânicos presentes em maior quantidade nesses solos. No solo com ureia e ureia revestida os maiores teores de nitrogênio amoniacal foram registrados no início e aos 38 dias de incubação devido à rápida solubilização das fontes minerais. Os teores de $\mathrm{N}^{-\mathrm{NH}_{4}}{ }^{+}$foram menores para solo de vinhedo e composto orgânico, no início e aos 38 dias de incubação, devido à aplicação superficial que diminuiu o contato solo e fertilizante orgânico e retardou a atividade microbiana sob 0 composto, somado ao fato que apenas $45,3 \%$ do $\mathrm{N}$ adicionado via composto estava na sua forma disponível (LORENSINI et al., 2014).

Os maiores valores de $\mathrm{NH}_{4}{ }^{+}$no solo sob vegetação nativa e com adubação orgânica encontrados no experimento, podem ser explicados devido a maior adição de componentes orgânicos nesses solos que após a mineralização resultou em maior disponibilidade de $\mathrm{N}$ ao longo do tempo. Além disso, no sistema orgânico o $\mathrm{N}$ adicionado poderia não estar em sua forma totalmente disponível ocorrendo a liberação de maneira gradual. O fertilizante orgânico fornece liberação gradual de N dependente do tipo de cadeia orgânica em que está inserido, podendo apresentar alta ou baixa labilidade (GROHSKOPF et al., 2015). Outro fator que pode ter influenciado maiores valores de $\mathrm{NH}_{4}{ }^{+}$no solo com adubação orgânica é a possível presença de inibidores da nitrificação biológica fornecido pelo material vegetal compostado. A inibição da nitrificação biológica é um processo onde as raízes de certas plantas suprimem a atividade de nitrificação do solo, por meio da produção e liberação de inibidores da nitrificação biológica, bloqueando a transformação de amônio em nitrato (SUBBARAO et al., 2015).

No tratamento convencional, a rápida solubilização das fontes minerais de $\mathrm{N}$ pode ter acarretado menores valores de $\mathrm{NH}_{4}{ }^{+}$nesse sistema. Fertilizantes minerais fornecem $\mathrm{N}$ mais rapidamente ao solo devido ao aumento da solubilidade físicoquímica dos seus grânulos (CORREA et al., 2016).

Os teores de fósforo assimilável foram maiores em cafeeiros submetidos ao manejo em transição para orgânico e convencional, diferenciando significativamente em relação ao solo de mata, o qual apresentou teor 12,7 vezes inferior em relação aos solos cultivados (Tabela 2).

Esses resultados diferem do encontrado por Velmourougane (2016), que detectou maior teor de fósforo em solo de cafeeiro sob sistema convencional em comparação ao sistema orgânico durante doze anos em Chettalli na Índia, devido à 
aplicação de fertilizantes fosfatados com alto teor deste nutriente no sistema convencional.

Avaliando o teor de fósforo disponível em área com sete anos de cultivo orgânico, área em transição com dois anos de cultivo orgânico e área de cultivo convencional em comparação com solo de mata no município de Areia - PB, Silva et al. (2015a) observaram maiores teores deste nutriente em solos do cultivo orgânico, seguido de sistema em transição, e sistema convencional e mata que apresentaram os menores valores. Os aumentos nos teores de $P$ no manejo orgânico e em transição estão relacionados com aplicação contínua de esterco e composto orgânico nesses sistemas. O menor valor encontrado no tratamento convencional ocorreu devido à ausência de práticas para manutenção da fertilidade do solo, como adição de matéria orgânica e reposição de nutriente. Já em solo de mata pode ser explicado pela baixa disponibilidade natural e alocação de nutrientes na vegetação (SILVA et al., 2015a).

Maiores valores de fósforo encontrados no manejo em transição para orgânico e convencional podem ser explicados devido à adubação com fontes fosfatadas que antecederam a implantação do experimento, verificada pelo teor alto do nutriente no solo na análise de solo (Quadro 1), e a manutenção por meio da adubação fosfatada no sistema convencional e por meio da aplicação do composto no sistema orgânico, que apesar de não apresentarem diferença significativa, o manejo orgânico apresentou teor de fósforo $19,37 \%$ maior que o tratamento convencional. $O$ aporte de material orgânico pode aumentar a disponibilização de $P$, por diminuir a energia de ligação dos fosfatos aos coloides inorgânicos e competir com os sítios de adsorção. Os menores valores observados para mata se devem às características dos solos de Cerrado, solos tropicais, principalmente mais intemperizados, como os Latossolos que apresentam predomínio de óxidos de ferro e alumínio que conferem alta adsorção de fosfatos (BEZERRA et al., 2015).

Analisando os dados de $\mathrm{C}_{\text {Lábil, }}$ não houve diferença significativa ao nível de $5 \%$ de probabilidade entre as médias para os tratamentos submetidos ao manejo em transição e convencional em comparação com a mata nativa (Tabela 2).

Os níveis de carbono no solo são influenciados pelo clima, textura e estrutura do solo, formas de uso e manejo do solo, taxa de deposição de resíduos e a intensidade dos processos de decomposição da matéria orgânica. O carbono lábil representa a fração mais lábil da matéria orgânica do solo que é sensível ao revolvimento do solo e mais facilmente mineralizada em condições de manejos não conservacionistas (ROSSI et al., 2016).

Avaliando cinco sistemas agroecológicos de produção em Seropédica - RJ, sendo eles sistema agroflorestal, com 10 anos de implantação; café a pleno sol, com 15 anos; café sombreado, com 15 anos; cultivo em aleias, com 10 anos de flemingia (Flemingia macrophylla) e com feijão (Phaseolus vulgaris var. vulgaris) e sistema plantio direto, com milho e berinjela, com 6 anos, Rossi et al. (2016) verificaram maiores teores de carbono orgânico particulado, que representa as frações lábeis da matéria orgânica, em cafeeiros agroecológicos sombreado e a pleno sol, explicado pelo maior tempo de manejo agroecológico do solo e o aporte de material orgânico pela espécie arbórea no cultivo sombreado e pela manutenção de vegetação na entrelinha no cultivo a pleno sol.

Estudando as frações oxidáveis da matéria orgânica em área de cerrado nativo, pastagem e sistema de plantio direto (SPD) com 3, 15 e 20 anos de 
implantação, em Montividiu - GO, Guareschi et al. (2013) verificaram aumento da fração lábil no SPD com 15 e 20 anos, sendo semelhante à vegetação de cerrado nativo e superior ao SPD com três anos e pastagem. Os menores valores observados em pastagem podem ser explicados pelo processo avançado de degradação desse solo e em SPD com três anos possivelmente ocorreu pelo fato de a área ter sido anteriormente cultivada com pastagem por 20 anos. Os maiores valores para SPD a 15 e 20 anos de implantação e cerrado nativo ocorreram devido à constante entrada de resíduos vegetais nesses sistemas que promoveram alterações no conteúdo da matéria orgânica.

A constante incorporação de resíduos orgânicos em solo cultivado com cafeeiro por meio da deposição de folhas sob o solo, incorporação de palha de café e o não revolvimento do solo, podem explicar a manutenção nos teores de $C_{\text {Lábil }}$ para sistema orgânico em transição e convencional semelhantes ao solo de mata nativa. O composto orgânico aplicado não alterou os teores de $C_{\text {Lábil }}$ no período de condução do experimento quando comparado ao tratamento convencional.

A maior atividade microbiana do solo foi verificada em solo sob vegetação nativa, diferindo significativamente dos tratamentos com adubação orgânica e convencional, os quais não diferenciaram entre si (Tabela 2).

Esses resultados diferem do encontrado em experimento com C. arabica L. em Chettalli na Índia, pois se verificou um aumento da respiração basal do solo em $15,4 \%$ e $8,6 \%$, nas camadas de $0-15$ e $15-30 \mathrm{~cm}$, respectivamente, em solo com sistema orgânico em comparação ao convencional, após doze anos de manejo (VELMOUROUGANE, 2016).

O uso de herbicidas também é um fator que pode interferir na atividade microbiana do solo, sendo a atrazina um dos produtos fitossanitários que mais interfere na composição da microbiota do solo ao longo do tempo (MORETTO et al., 2017). Os baixos valores observados no trabalho quanto à atividade microbiana do solo sob adubação orgânica podem ter ocorrido devido à deriva de produtos fitossanitários aplicados no manejo convencional.

Algumas frações da matéria orgânica apresentam maior persistência no solo como a fração humina, que apresenta maior dificuldade de degradação (SILVA et al., 2015a). Os componentes presentes no composto podem ter interferido na sua degradação no solo, provavelmente devido a presença de componentes de difícil degradação que não proporcionou incrementos na atividade microbiana para solos sob adubação orgânica.

Para a condutividade elétrica verificou-se diferenças entre os tratamentos, sendo o solo de mata nativa o que apresentou menor valor médio $\left(33,33 \mu \mathrm{S} \mathrm{cm}{ }^{-1}\right)$, se comparado ao cafeeiro em transição para orgânico e convencional, os quais não diferiram significativamente entre si (Tabela 3). Os maiores valores de condutividade elétrica nos solos cultivados podem ser explicados devido à aplicação de fertilizantes na área que antecederam a implantação do experimento. O período de condução do experimento não permitiu observar diferenciação entre os tratamentos com manejo em transição e convencional. 
TABELA 3- Médias de pH e condutividade elétrica (CE) no solo sob manejo de transição para orgânico e convencional comparado com mata nativa

\begin{tabular}{ccc}
\hline Tratamento & $\mathbf{C E}\left(\boldsymbol{\mu S \mathbf { ~ c m } ^ { - 1 } )}\right)$ & $\mathbf{p H}$ \\
\hline MATA & $33,33 \mathrm{~b}$ & $5,03 \mathrm{~b}$ \\
T1 & $71,70 \mathrm{a}$ & $6,12 \mathrm{a}$ \\
T2 & $101,23 \mathrm{a}$ & $6,02 \mathrm{a}$ \\
T3 & $89,21 \mathrm{a}$ & $6,21 \mathrm{a}$ \\
T4 & $81,89 \mathrm{a}$ & $6,07 \mathrm{a}$ \\
\hline CV $(\%)$ & 6,03 & 4,86
\end{tabular}

Médias seguidas pela mesma letra não diferem significativamente entre si a $5 \%$ de probabilidade pelo teste de Scott-Knott.

Em experimento com $C$. arabica L. submetido ao manejo convencional e orgânico durante doze anos, em Chettalli na Índia, o sistema de manejo convencional apresentou maiores valores de condutividade elétrica, nas profundidades de 0-15 e 15-30 cm, quando comparado ao manejo orgânico. O aumento da condutividade elétrica no sistema convencional é uma indicação de acumulação de sais devido o uso de fertilizantes (VELMOUROUGANE, 2016).

Observou-se diferença significativa para acidez do solo pelo teste de ScottKnott ao nível de $5 \%$ de probabilidade, entre o solo de mata nativa e os solos cultivados (Tabela 3). Foram encontrados maiores valores de $\mathrm{pH}$ em solo sob manejo de transição para orgânico e convencional em relação à mata nativa que apresentou maior acidez média ( $\mathrm{pH} 5,03$ ), podendo ser explicado pela correção do solo empregada nesses sistemas. Resultados semelhantes foram verificados por Guimarães et al. (2013), os quais detectaram maior acidez média em solo de mata nativa quando comparado ao cafeeiro orgânico e convencional em lavoura cultivada com Catuaí Vermelho no município de lúna-ES.

\section{CONCLUSÕES}

O manejo em transição para orgânico proporcionou maior disponibilização de nitrogênio amoniacal em relação ao manejo convencional do cafeeiro.

Os maiores teores de fósforo foram encontrados na área cultivada, o que sugere que esses manejos foram eficientes em manter os teores do nutriente no sistema.

Os valores de $\mathrm{C}_{\text {Lábil }}$ foram semelhantes ao do solo de mata para os dois sistemas de manejo.

Não houve alteração na atividade microbiana para manejo em transição para orgânico e convencional, sendo a maior atividade encontrada em solo de mata.

Maiores valores de $\mathrm{pH}$ e condutividade elétrica foram detectados nos solos cultivados.

\section{REFERÊNCIAS}

AZEVEDO JUNIOR, R. R; SANTOS, J. B; BARETTA, D; RAMOS, A. C; CARDOSO, E. J. B. N. Chemical and microbiological soil properties in organic and conventional management systems of Coffea arabica L. Journal of Plant Nutrition. v. 40, n. 14, p.2076-2086, 19 jul. $2017 . \quad$ Disponível em: https://www.tandfonline.com/doi/abs/10.1080/01904167.2017.1346128 DOI: https://doi.org/10.1080/01904167.2017.1346128 
BEZERRA, R. P. M.; LOSS, A.; PEREIRA, M. G.; PERIN, A. Frações de fósforo e correlação com atributos edáficos sob sistemas de plantio direto e integração lavoura-pecuária no Cerrado Goiano. Semina: Ciências Agrárias, Londrina, v. 36, n. 3 , p.1287-1306, jun. $2015 . \quad$ Disponível em: https://www.redalyc.org/articulo.oa?id=445744148010 DOI: $\quad 10.5433 / 1679$ 0359.2015v36n3p1287

BLAIR, G.J.; LEFROY, R.D.B.; LISLE, L. Soil carbon fractions based on their degree of oxidation, and the development of a carbon management index for agricultural systems. Australian Journal of Agricultural Research, v. 46, p.1459-1466, 1995. Disponível em: https://www.publish.csiro.au/CP/AR9951459 DOI: https://doi.org/10.1071/AR9951459

BORRELLA, I.; MATAIX, C.; CARRASCO -GALLEGO, R. Smallholder farmers in the speciality coffee industry: opportunities, constraints and the businesses that are making it possible. IDS Bulletin, Brighton, v. 46, n. 3, p. 29-44, 2015. Disponível em: https://onlinelibrary.wiley.com/doi/abs/10.1111/1759-5436.12142 https://doi.org/10.1111/1759-5436.12142

COMPANHIA NACIONAL DE ABASTECIMENTO. Acompanhamento da safra brasileira de café, v. 6 - Safra 2020, n.1- Primeiro levantamento, Brasília, p. 1-62, jan. 2020. Disponível em: https://www.conab.gov.br/info-agro/safras/cafe/boletim-dasafra-de-cafe Acesso em 10/02/2020.

CORREA, J. C.; GROHSKOPF, M. A.; NICOLOSO, R. Da. S.; LOURENÇO, K. S.; MARTINI, R. Organic, organomineral, and mineral fertilizers with urease and nitrification inhibitors for wheat and corn under no-tillage. Pesquisa Agropecuária Brasileira, v. 51, n. 8, p.916-924, 2016. Disponível em: http://www.scielo.br/scielo.php?pid=S0100-204X2016000800916\&script=sci_arttext.

DOI: http://dx.doi.org/10.1590/S0100-204X2016000800003

DIONÍSIO, J. A.; PIMENTEL, I. C.; SIGNOR, D. Respiração microbiana: Respiração basal do solo. In: DIONÍSIO, J. A.; PIMENTEL, I. C.; SIGNOR, D.; PAULA, A. M. De.; MACEDA, A.; MATTANA, A. L. Guia prático de biologia do solo. Curitiba: Sociedade Brasileira de Ciência do Solo/ Nepar, Cap. 12, p. 72-75, 2016.

FERREIRA, D. F. Sisvar: um sistema computacional de análise estatística. Ciência e Agrotecnologia. v. 35, n. 6, pp.1039-1042, 2011. Disponível em http://www.scielo.br/scielo.php?pid=S141370542011000600001\&script=sci_abstract \&tlng=pt DOI: https://doi.org/10.1590/S1413-70542011000600001

GUARESCHI, R. F.; PEREIRA, M. G.; PERIN, A. Oxidizable carbon fractions in Red Latosol under different management systems. Revista Ciência Agronômica, Fortaleza, v. 44, n. 2, p.242-250, 2013. Disponível em: http://www.scielo.br/scielo.php?pid=S1806-66902013000200005\&script=sci_arttext DOI: http://dx.doi.org/10.1590/S1806-66902013000200005 
GUIMARÃES, G. P.; MENDONÇA, E. S.; PASSOS, R. R.; ANDRADE, F. V.; MACHADO, R. V. Avaliação da qualidade do solo e de cafeeiros em propriedade familiar do Território do Caparaó-ES. Revista Brasileira de Agroecologia, v. 3, n. 8, p.236-246, 2013. Disponível em: http://revistas.abaagroecologia.org.br/index.php/rbagroecologia/article/view/13347 Acesso em $10 / 02 / 2020$

GROHSKOPF, M.A.; CASSOL, P.C.; CORREA, J.C.; MAFRA, M.S.H.; PANISSON, $J$ J. Organic nitrogen in a Typic Hapludox fertilized with pig slurry. Revista Brasileira de Ciência do Solo, v.39, p.127-139, 2015. Disponível em: http://www.scielo.br/scielo.php?pid=S0100-06832015000100127\&script=sci_arttext. DOI: http://dx.doi.org/10.1590/01000683rbcs20150080

LOPES, P. R.; ARAÚJO, K. C. S; LOPES, I. M.; RANGEL, R. P.; SANTOS, N. F. F.; KAGEYAMA, P. Y. Uma análise das consequências da agricultura convencional e das opções de modelos sustentáveis de produção - agricultura orgânica e agroflorestal. Revista Espaço de Diálogo e Desconexão, Araraquara, v. 8, n.1 e 2, 2014. Disponível em: https://periodicos.fclar.unesp.br/redd/article/view/6912/5605.

LORENSINI, F.; CERETTA, C.A.; BRUNETTO, G.; CERINI, J.B.; LOURENZI, C.R. et al. Disponibilidade de nitrogênio de fontes minerais e orgânicas aplicadas em um Argissolo cultivado com videira. Revista Ceres, Viçosa, v. 61, n. 2, p. 241-247, 2014. Disponível em: 737X2014000200012\&script=sci_arttext. DOI: http://dx.doi.org/10.1590/S0034$737 X 2014000200012$

MARTINEZ-SALGADO, M. M.; GUTIERREZ-ROMERO, V.; JANNSENS, M.; ORTEGA-BLU, R. Biological soil quality indicators: a review. In: MENDEZ-VILAS, A. (Ed.). Current research, technology and education topics in applied microbiology and microbial biotechnology. [Badajoz]: Formatex Research Center, p. 319-328, 2010.

MENDES, I. C.; SOUSA, D. M. G. De.; REIS JUNIOR, F. B. Dos. Bioindicadores de qualidade de solo: dos laboratórios de pesquisa para o campo. Cadernos de Ciência \& Tecnologia, Brasília, v. 32, p.185-203, 2015. Disponível em: https://seer.sct.embrapa.br/index.php/cct/article/view/23311. DOI: http://dx.doi.org/10.35977/0104-1096.cct2015.v32.23311

MORETTO, J. A. S.; ALTARUGIO, L. M.; ANDRADE, P. A.; ANDREOTE, F. D. ; STEHLING, E. G. Changes in bacterial community after application of three different herbicides. Fems Microbiology Letters, v. 364, n. 13, fnx113, 2017. Oxford University Press (OUP). Disponível em: https://academic.oup.com/femsle/article/364/13/fnx113/3861255 DOI: http://dx.doi.org/10.1093/femsle/fnx113

PASQUINI, C.; DE FARIA, L. C. Flow-injection determination of ammonia in kjeldahl digests by gas diffusion and conductometry. Analytica Chimica Acta, v. 193, p.1927 , 1987.

Disponível

em: 
https://www.sciencedirect.com/science/article/pii/S0003267000861346

DOI: https://doi.org/10.1016/S0003-2670(00)86134-6

ROSSI, C. Q.; PEREIRA, M. G.; MOURA, O. V. T. De.; ALMEIDA, A. P. C. De. Vias de formação, estabilidade e características químicas de agregados em solos sob sistemas de manejo agroecológico. Pesquisa Agropecuária Brasileira, v. 51, n. 9, p.1677-1685, set. 2016. Disponível em: http://www.scielo.br/scielo.php?pid=S0100204X2016000901677\&script=sci_arttext DOI: http://dx.doi.org/10.1590/s0100$204 \times 2016000700068$

SHANG, C.; TIESSEN, H. Organic matter lability in tropical Oxisol: evidence from shifting cultivation, chemical oxidation, particle size, density, and magnetic fractionations. Soil Science, v.162, p.795-807, 1997. Disponível em: https://journals.Iww.com/soilsci/Abstract/1997/11000/ORGANIC_MATTER_LABILITY _IN_A_TROPICAL_OXISOL_.4.aspx. Acesso em: 10/02/2020

SILVA, D. De. F.; ANDRADE, C.L; SIMEONE, M.L.F.; AMARAL, T.A.; CASTRO, L.A;. MOURA, B.F.; Análise de nitrato e amônio em solo e água. Embrapa Milho e Sorgo-Documentos (INFOTECA-E), 55p, 2010. Disponível em: https://www.embrapa.br/milho-e-sorgo/busca-de-publicacoes/-

/publicacao/busca/An\%C3\%A1lise\%20de\%20Nitrato\%20e\%20Am\%C3\%B4nio\%20e m\%20Solo\%20e\%20\%C3\%81gua?. Acesso em 10/02/2020.

SILVA, G. F.; SANTOS, D.; SILVA, A. P. Da.; SOUZA, J. M. De. Indicadores de qualidade de solo sob diferentes sistemas de uso na Mesorregião do Agreste Paraibano. Revista Caatinga, Mossoró, v. 28, n. 3, p.25-35, set. 2015a. Disponível em: https://www.redalyc.org/pdf/2371/237141066003.pdf. Acesso em :10/02/2020

SILVA, V. M. De.; TEIXEIRA, A. F. R.; REIS, E. F. Dos.; BENASSI, A. C.; MENDONÇA, E. De. S. Estoques de Carbono e Nitrogênio e Densidade do Solo em Sistemas de Adubação Orgânica de Café Conilon. Revista Brasileira de Ciência do Solo, v. 39, n. 5, p.1436-1444, out. 2015b. Disponivel em: http://www.scielo.br/scielo.php?pid=S010006832015000501436\&script=sci_arttext\&tl ng=pt. DOI: http://dx.doi.org/10.1590/01000683rbcs20140520

SUBBARAO, G. V.; YOSHIHASHI T.; WORTHINGTON, M.; NAKAHARA, K.; ANDO, Y. et al. Suppression of soil nitrification by plants. Plant Science, v. 233, p.155-164, 2015.

Disponível

em: https://www.sciencedirect.com/science/article/pii/S016894521500031X DOI: https://doi.org/10.1016/j.plantsci.2015.01.012

VELMOUROUGANE, K. Impact of Organic and Conventional Systems of Coffee Farming on Soil Properties and Culturable Microbial Diversity. Scientifica, v. 2016, p.1-9, 2016 Disponiel em: https://www.hindawi.com/journals/scientifica/2016/3604026/. DOI: https://doi.org/10.1155/2016/3604026 